\title{
What prospects for an X-ray laser?
}

WHAT are the chances that one day there will be machines capable of producing short but intense pulses of virtually monochromatic $X$ rays, for all the world like lasers that function at the shortwavelength end of the spectrum? And if such machines can be built, what purposes will they serve? The notion that a machine with such a specification might be the basis of yet another military weapon will no doubt quickly capture the imagination of newspaper-headline writers. In reality, of course, the Earth's atmosphere is as opaque, more or less gram for gram, as are other materials. For those who brood about "death rays" for the benefit of science fiction literature, what matters (or should matter) is not the wavelength of the radiation but the amount of energy that can be delivered by a radiation beam and the efficiency with which it is absorbed by the target. Reflection will also show that the present attempts to develop X-ray lasers, centred as they are on machines that differ very little from highenergy particle accelerators, suggest that X-ray lasers - if they can be built - will hardly be the kinds of portable devices of which generals and their staffs are fond. In other roles, however, and especially in strictly scientific applications, devices of this kind, however cumbersome, would be of great benefit. So what are the chances that they will eventually be constructed?

This question has obviously perplexed those within the European Science Foundation who have been concerned with the project to plan a European facility for synchrotron radiation. At the general assembly of the foundation last week in Strasbourg, those responsible for this project, which is essentially a scheme for building an electron storage ring operating at $3 \mathrm{GeV}$ and designed to produce copious amounts of synchrotron radiation, let it be known that they are undeterred by the chance that an X-ray laser may eventually make their own project unnecessary. In this phlegmatic view, the planning group is surely on safe ground. The chances that it will be possible to construct machines operating as lasers in the X-ray range within the lifetime of the proposed European synchrotron machine are small. Further ahead, however, there is a reasonable chance that something may be possible.

The starting point for this speculation is the wave of excitement generated in the past few years by the concept of the "freeelectron laser", an innovation largely due to Dr J. Madey at Stanford University in 1976 (Elias, L.R. et al. Phys. Rev. Lett. 36, $717 ; 1976)$. The working principle of these devices is a curious amalgam of old-fashioned 1940s technology and the more recent sophistication of the particle-beam technologists (see J.N. Elgin Nature News and Views, 1 May 1980, and references therein). Suppose a beam of electrons travelling along a tube is subjected to a sequence of transverse magnetic fields, alternately in one direction and the opposite. At each step, the electrons (accelerated as they will be along the third cartesian axis) will radiate electromagnetic energy. As Maxwell's equations have it, a beam of electrons processed in this way should be a source of a radiation beam directed along the axis of the system. Moreover, the wavelength of the radiation should be determined to a first approximation only by the energy of the electrons and the geometrical periodicity of the magnetic field. The more frequent the alternation of the direction of the magnetic field along the axis of the beam, the shorter the wavelength of the radiation, which is (or should be) related inversely to the square of the energy of the electrons. The planning of the proposed European synchrotron radiation facility is based almost entirely on the notion that a circulating beam of stored electrons should be subjected to a sequence of such corrugated magnetic fields, called "wigglers" in the trade.

$0028-() 836 / 80 / 470209-01 \$ 01.00$
The novel development at Stanford University was the recognition that a beam of electrons travelling through a corrugated magnetic field should also behave as if it were the working material of a laser. In other words, if an electron beam traversing a wiggling magnetic field is also exposed to radiation of the frequency characteristic of the geometry and the electron energy, radiation at that same frequency will be stimulated and, if there is a pair of mirrors somewhere, energy from the mechanical motion of the electrons will be converted into a beam of radiation of wavelength determined in advance only by geometry and electronic energy (with the actual strength of the magnetic field as a second approximation correction). So, would-be designers of $\mathrm{X}$-ray lasers will say, why not go the whole hog, pushing the electron energy as high as possible but reducing the geometrical wavelength of the alternation of the wiggling magnetic fields until the predicted wavelength is in the $\mathrm{X}$-ray region?

There seems little doubt that lasers working on this principle will be designed and built in the years ahead. For the time being, however, the wavelengths at which free-electron lasers are being designed or played with are modestly long. The first experiments at Stanford University demonstrated laser action at $10.6 \mu \mathrm{m}$, well into the infrared. Although there are more conventional types of lasers which function in this region of the spectrum, the prospect that the wavelength of radiation from a free-electron laser may be adjusted simply by changing the energy of the electron beam suggests that devices built on such a principle should be more 'tuneable' than more conventional infrared lasers. The immediate prize in the sights of those now developing this new technology is, however, that of being able to construct lasers that operate in the ultraviolet. The potential benefits of such devices are self-evident. For the first time, it would, for example, be possible to make precise studies of several photochemical processes at present inaccessible to observation. The snags are unfortunately also clear. Free-electron lasers are likely to be less efficient at increasing energies (although much depends on the geometry) while technical problems of arranging for a geometrically rapid corrugation of a magnetic field entail the complications of handling superconducting magnets. This part of the game, nevertheless, may be well worth the candle because of the potential usefulness of ultraviolet lasers in the separation of uranium isotopes or the stimulation of thermonuclear fusion.

The potential of free-electron lasers seems to have been clearly recognized in the United States, where much technical development is under way. The best known plan is that of the Brookhaven National Laboratory on Long Island, where a $700-\mathrm{MeV}$ storage ring will be commissioned next year with the intention of producing laser radiation in the optical and near-ultraviolet in 1982. Most other projects, in which industrial companies such as TRW Incorporated and Bell Telephone Laboratories have taken an interest, appear to be aimed at exploring still inaccessible parts of the infrared spectrum. Given the present limitations of even superconducting magnet design, it is inevitable that people should be forced to use beams of electrons with energies approaching 1 or more GeV. But these, of course, are early days. With any luck, free-electron lasers should be operating in the ultraviolet within the next few years. There after, it should be relatively easy to decide how the technology might be pushed still further. By the end of the decade, soft X-ray laser sources, however inefficient, should be in operation. The hard X-ray source that will make possible direct measurement of atomic and molecular energy levels will be further ahead, but not out of reach. 